\title{
Application of nanogel systems in the administration of local anesthetics
}

This article was published in the following Dove Press journal:

Local and Regional Anesthesia

30 August 2010

Number of times this article has been viewed

\section{Jeremy PK Tan' \\ Maureen BH Tan ${ }^{2}$ \\ Michael KC Tam ${ }^{3}$}

'Institute of Bioengineering and Nanotechnology, Agency for Science Technology and Research, Singapore; ${ }^{2}$ Institute of Materials Research and Engineering, Agency for Science Technology and Research, Singapore; ${ }^{3}$ Department of Chemical Engineering, University of Waterloo, Waterloo, Ontario, Canada
Correspondence: Jeremy PK Tan and Michael KCTam

Institute of Bioengineering and Nanotechnology, Agency for Science Technology and Research, 3I Biopolis Way, The Nanos, \#04-0I, Singapore; Department of Chemical Engineering, University of Waterloo, 200 University Avenue West, Waterloo, Ontario, Canada Email pktan@ibn.a-star.edu.sg; mkctam@uwaterloo.ca
Abstract: Nanogels are robust nanoparticles that could be used to deliver active drug compounds in controlled drug delivery applications. This review discusses the design, synthesis, loading, and release of local anesthetics using polymeric nanoparticles produced via various types of polymerization techniques. The strategy of using layer-by-layer approach to control the burst release of procaine hydrochloride (PrHy; a local anesthetic drug of the amino ester group) is described and discussed.

Keywords: nanogels, nanoparticles, active drug compounds, local anesthetics, polymeric nanoparticles, procaine hydrochloride (PrHy)

\section{Introduction}

One of the top priorities in therapeutics is pain control and in spite of the recent advances in clinical investigation of new therapeutic agents, pain relief is still a significant challenge. The reasons include difficulties of correctly evaluating pain, underestimation of patients' pain, misconceptions about analgesic use and side effects, gaps in pain management process, and lack of acute pain service. ${ }^{1}$ Local anesthetics (LAs) are among the different classes of pharmacological compounds used to eliminate pain. LAs are small molecules that could be easily eluted from the site of administration, thus limiting the analgesic property.

Cocaine is the first compound that was used as a "numbing agent" in the early 1500 s. However, it is usually associated with the central nervous system and cardiovascular system toxicity. Presently, most of the synthetic LAs are structurally related to cocaine but with reduced toxicity. LA works by cutting the signal of nerve impulses in nerve cell membranes through the shutting of voltage-gated $\mathrm{Na}^{+}$channels. The degree of numbness from a certain concentration will depend on how the nerve is stimulated and the resting membrane potential. ${ }^{2}$ Clinical LAs belong to one of the two classes: amino esters (Figure 1A) and amino amide (Figure 1B). Amino ester LAs were developed in early 1900s. Ester-based LAs are hydrolyzed and inactivated by plasma esterase, primarily plasma cholinesterase. Therefore, they are always associated with low potency, slow onset, and short therapeutic duration. ${ }^{2}$ Amino amide LAs became popular and many of this class of drugs were developed by 1970s. Amide-based LAs are degraded by hepatic endoplasmic reticulum, leading to a slower removal from the body and higher concentration in the plasma. With the introduction of bupivacaine, accidental overdose was often fatal and responded poorly to conventional resuscitation. ${ }^{2,3}$ This had led to a search for long-acting LAs with improved therapeutic margins and reduced toxicity. 


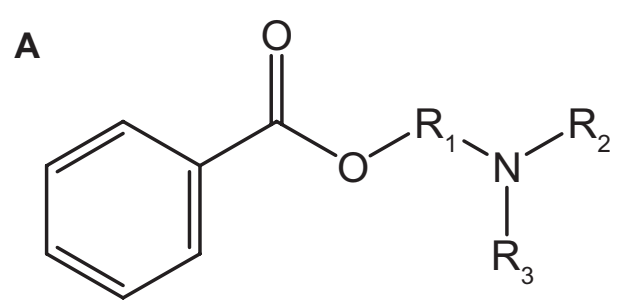

Figure I Chemical structure for (A) amino ester and (B) amino amide.

Trachez et al and Zapata-Sudo et al demonstrated that S(-) enantiomer of bupivacine is more potent ${ }^{4}$ and less toxic ${ }^{5}$ than $\mathrm{R}(+)$ enantiomer leading to the selection of $\mathrm{S}(-)$ enantiomers of ropivacine and levobupivacine as safer alternatives to bupivacine.

High toxicity of LA due to over dosage and strong clinical requirements have triggered the interest in the field on controlled release of LA. One of the primary objectives in the design of a controlled release system is the controlled delivery of therapeutics to the site with an optimal release rate. From here, two major areas have evolved: (1) design of good carriers to protect and deliver the therapeutics to the site of action and (2) development of a sustained drug formulation for a slow and long lasting effect. ${ }^{6}$ The release of these drugs will be controlled by desorption, diffusion, erosion, or a combination of these factors. ${ }^{7}$ One approach to prolong the effect of analgesia is to complex the LA with drug delivery vehicles. The larger carriers will remain at the injection site for a longer period of time with a gradual release of the LA. Liposomes, ${ }^{8-11}$ cyclodextrins, ${ }^{12}$ microspheres, ${ }^{13-15}$ hydrogels, ${ }^{16}$ and nanospheres ${ }^{6,17-20}$ could encapsulate the LA for controlled delivery, prolonged anesthetic effect, and reduced toxicity. From these various types of controlled delivery systems, depending on the end-use requirements, the most popular choice is nanospheres followed by microspheres and hydrogels. ${ }^{21}$

In this review, different methods of nanogel synthesis and an overview of drug release from nanogel-based delivery vehicles will be described and discussed.

\section{Synthesis technique}

Current approaches used for the preparation of nanogels can be classified into four categories: (1) polymerization of monomers in a homogeneous phase or in a microscale or nanoscale heterogeneous environment; (2) physical self-assembly of interactive polymers; (3) cross-linking of preformed polymers; and (4) template-assisted nanofabrication of nanogel particles. In this section, we will discuss these methods, where the synthesis technique being employed is typically

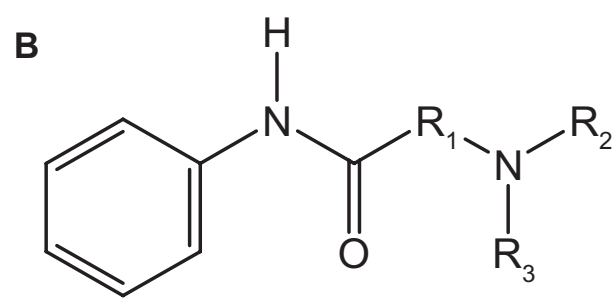

dictated by the desired application and types of study being performed.

Surfactant-free emulsion polymerization or precipitation polymerization is a versatile technique for synthesizing stimuli-responsive nanogels. Nanogels synthesized by this method are formed by homogeneous nucleation of water-soluble monomers, which results in the formation of a colloidal suspension of the growing polymer, and the charge imparted by the initiator stabilizes the gels. This method is versatile from the standpoint of particle size control. To synthesize smaller nanogels, the precursor particles must be stabilized earlier in the reaction, where an ionic surfactant can be added to impart colloidal stability. Similarly, larger particles can be obtained by decreasing the surfactant concentration. ${ }^{22}$ Peppas and coworkers ${ }^{23}$ synthesized a suspension of nanospheres composed of poly (ethylene glycol) (PEG)-grafted poly (methacrylic acid) (PMA) in water using the precipitation polymerization method. Thermoresponsive poly ( $N$-isopropylacrylamide) (PNIPAM) nanogels with narrow size distribution were first synthesized by Pelton and Chibante, ${ }^{24,25}$ where all the monomers and cross-linker are dissolved in water.

Precipitation polymerization is best for materials that are hydrophobic, so that they can readily attach to the collapsed precursor particle and is useful only for materials that are stable at high temperatures, and hence it cannot be used to incorporate biological macromolecules. Thus, if a hydrophilic comonomer is to be copolymerized, only a certain weight percentage can be incorporated. ${ }^{22}$ To overcome these drawbacks, inverse microemulsion polymerization is used, where an aqueous solution of all the monomers is added to an appropriate amount of oil and surfactant to produce a thermodynamically stable microemulsion. Various drugs, DNA, and cells are physically incorporated into these aqueous microemulsions, which are then cross-linked with appropriate cross-linking agents. The resulting cross-linked nanogels are prepared by dispersion in organic solvents, and then purified by precipitation, centrifugation, washing (usually with organic solvents, such as isopropanol), and lyophilization. Several reports have 
demonstrated the synthesis of hydrophilic or water-soluble particles comprising of poly (2-hydroxyethyl methacrylate) (PHEMA), ${ }^{26}$ polyacrylic acid (PAA), ${ }^{27}$ polyacrylamide (PAAm), ${ }^{26}$ PNIPAM, ${ }^{28-30}$ and polyaniline. ${ }^{31}$ These nanoparticles can be either hollow and zwitterionic or core-shell nanocapsules with hydrophobic shell and hydrophilic interior. Labile bonds are frequently introduced into the nanogels during polymerization to make them degradable and facilitate drug release. ${ }^{32-36}$ Matyjaszewski and coworkers ${ }^{35,36}$ used the atom transfer radical polymerization (ATRP) performed in an inverse microemulsion system to synthesize stable biodegradable nanogels comprising of water-soluble polymers with a disulfide-functionalized cross-linker.

Nanogels can also be formed using the physical selfassembly of polymers involving a mixture of amphiphilic copolymer and a therapeutic agent in an organic solvent via membrane dialysis or from direct complexation between cationic copolymers and genes or proteins in an aqueous solution through noncovalent interactions, such as Van der Waals, hydrogen bonding, stereocomplexation, charge transfer, and polyelectrolyte complexation. During the self-assembly process, small molecular drugs and macromolecular therapeutics, such as genes and proteins, can be incorporated into the micellar nanostructures. The most widely used selfassociating polymers are amphiphilic block copolymers that possess hydrophobic and hydrophilic blocks that can self-assemble in selective solvents to form submicron-size micelles (nanogels). These amphiphilic polymers can be synthesized via living radical polymerization (LRP) techniques (eg, ATRP, reversible addition - fragmentation chain transfer [RAFT], and nitroxide-mediated polymerization [NMP]). The micellization of amphiphilic block copolymers can be tailored by changing the block nature, block lengths, composition, or architecture. ${ }^{37-39}$ In addition, external parameters, ie, change in temperature or solvent or addition of additives, such as electrolytes and cosolvents, provide extra degrees of freedom to tune the micellar behavior of the amphiphilic block copolymers. ${ }^{40-44}$ Moreover, the chemistry of the surface can be manipulated for the conjugation of a targeting signal that can recognize specific tissues or cells. The functionalities of the core of nanogels can be tuned using specific chemical approaches to improve the drug-loading capacity. The molecular compositions of the copolymers used to form the nanogels can be controlled through LRP approaches to achieve nanostructures with narrow size distribution.

In addition to the above two polymerization methods, covalent cross-linking of preformed polymer chains provides excellent opportunities for producing functional nanogels with large pore sizes for drug delivery. ${ }^{45}$ In particular, it was used to synthesize the first cross-linked cationic nanogel for polynucleotide delivery. ${ }^{46}$ In this case, a doubly activated PEG was conjugated to a branched polyethylenimine (PEI) in an oil in water emulsion (dichloromethane in water) followed by evaporation of the solvent in vacuo and maturation of the nanogel in an aqueous solution. Cationic PEI containing nanogels of 80-200 nm diameter were also obtained by the photo-Fenton reaction in aqueous media. ${ }^{47,48}$ Small (40-45 nm), nontoxic cross-linked pullulan nanogels were prepared in the reverse micellar system (aerosol OT/ hexane). ${ }^{49,50}$ The cross-links that connect the polymeric chains in such nanogels can be made degradable to reduce their toxicity. For example, a biodegradable segmented PEI connected by disulfide linkers was used to prepare cationic nanogels for polynucleotide delivery, ${ }^{51}$ and hyaluronic acid (HA) nanogels containing biodegradable disulfide linkages were prepared by the inverse water in oil emulsion method. ${ }^{52}$ An interesting type of cross-linked nanogels containing DNA was obtained by mixing thiol-functionalized 6-arm-branched PEG and DNA in dimethyl sulfoxide, which produced particles of $100 \mathrm{~nm}$ diameter and subsequently cross-linked by oxidation to obtain DNA-loaded biodegradable nanogels. ${ }^{53}$

Combining the controlled self-assembly of polymeric micelles and cross-linking techniques provided opportunities for the control of the spatial distribution of polymeric chains at the nanolength scale. For example, Wooley and coworkers ${ }^{54-58}$ have chemically cross-linked the shell layers of polymeric micelles to obtain various shell-cross-linked nanoparticles with morphologies, such as spheres, rods, and even toroids. In another study, PEI was cross-linked to micelles of doubly activated Pluronic ${ }^{\circledR}$ triblock copolymer in aqueous solution to produce nanogels with a hydrophobic poly ( $p$-phenylene oxide) (PPO) core surrounded by a swollen cross-linked PEI and a PEG shell. ${ }^{59}$ Lee et $\mathrm{al}^{60}$ photo-cross-linked the polymeric micelles of poly(D,Llactic acid)- $b$-PEG- $b$-poly(D,L-lactic acid) with acrylate end groups, resulting in the formation of nanogels containing selfassembled hydrophobic domains of micelles with insoluble poly(D,L-lactic acid) cores, which could be loaded with a hydrophobic anticancer drug.

Finally, DeSimone and coworkers ${ }^{61}$ developed a novel imprint photolithographic technique, particle replication in nonwetting templates (PRINT), for the fabrication of nanogels. They used nonwetting elastomeric molds of a low-surface-energy perfluoropolyether network prepared on patterned silicon templates by photochemical cross-linking of dimethacrylate-functionalized perfluoropolyether 
oligomers. The nonwetting molds eliminate the formation of a residual interconnecting film between the molded objects, thus producing monodispersed, shape-specific nanoparticles from an extensive array of organic precursors. This method enables strict control over the particle size, shape, composition, and surface functionality and permits the loading of delicate cargos, including pharmaceutical drugs and biomacromolecules. For example, monodispersed 200-nm-diameter PEG-based swellable particles were fabricated with the PRINT method by UV-induced copolymerization of several vinyl monomers, such as PEG triacrylate, PEG monomethyl ether monomethacrylate, and $p$-hydroxystyrene. ${ }^{62}$

\section{Nanogel systems as carriers}

Parenteral route is the only approach to administer drugs, as this will ensure the quickest onset of the therapeutic product. From the work of Lee et al, ${ }^{63}$ injections made with nanogels are less painful when compared with cosolventbased formulation. High blood circulation time could be achieved due to the size of the nanogels, which is very important to extend the therapeutic efficacy of the LA. Nanogels exhibit excellent thermodynamic stability, high solubilization capacity, low viscosity, and ability to withstand sterilization techniques, and these properties make nanogels as interesting delivery systems. ${ }^{64}$ Most importantly, after the encapsulation of LA into the nanospheres, the toxicity effect is greatly reduced as shown by the work of Moraes et al using poly(DL-lactide-co-glycolide) nanospheres encapsulated with either bupivacaine ${ }^{17}$ or ropivacaine. ${ }^{18}$ In both the cases, after encapsulation of the LA, there was a significant improvement in the viability of $3 \mathrm{~T} 3$ cells (Figure 2).

\section{Drug - nanogel interactions}

There are 2 goals that should be satisfied in the design of a delivery system: ${ }^{65}$ (1) efficient binding of drugs to the polymeric matrix and release of the drugs in a controlled manner and (2) ability to release through a local or externally applied trigger by changing the binding affinity between the drug and the polymeric matrix. Since most of the LAs possess secondary or tertiary amines, electrostatic interaction is the dominant driving force, depending on the $\mathrm{pH}$ of the solution and $\mathrm{pKa}$ of the LA. Therefore, a clear understanding on the interaction between the LA and the nanogel is critical for the development of an efficient drug delivery system. Procaine hydrochloride (PrHy; a LA drug of the amino ester group) loaded into methacrylic acid-ethyl acrylate (MAA-EA) nanogels through hydrophobic and hydrogen bonding (Figure 3) showed a higher release at a higher pH (Figure 4). ${ }^{66}$ At a higher $\mathrm{pH}$, the acid on the nanogels will be deprotonated, thus increasing the osmotic pressure and swelling the nanogels. This will promote the release of PrHy through the increase in porosity. ${ }^{20}$ Different kinds of interactions will result in a different release profile, which will be important for the design of a delivery carrier, where drug release can be triggered by changing the $\mathrm{pH}$. First, the results demonstrated the viability of tuning the release profiles of drug from a $\mathrm{pH}$-responsive nanogel system. The proportion of drug released can be controlled by manipulating the $\mathrm{pH}$ of the environment. Hence, it is possible to design a $\mathrm{pH}$-dependent gradient release drug delivery system, such that the active drugs could be released from the carrier in different regions of the physiological environment that possess different $\mathrm{pH}$ conditions. This will extend the therapeutic range of active drugs and ensure that the targeted areas receive the right dosage of drugs. Second, the interaction between the drug and delivery carriers controls
A

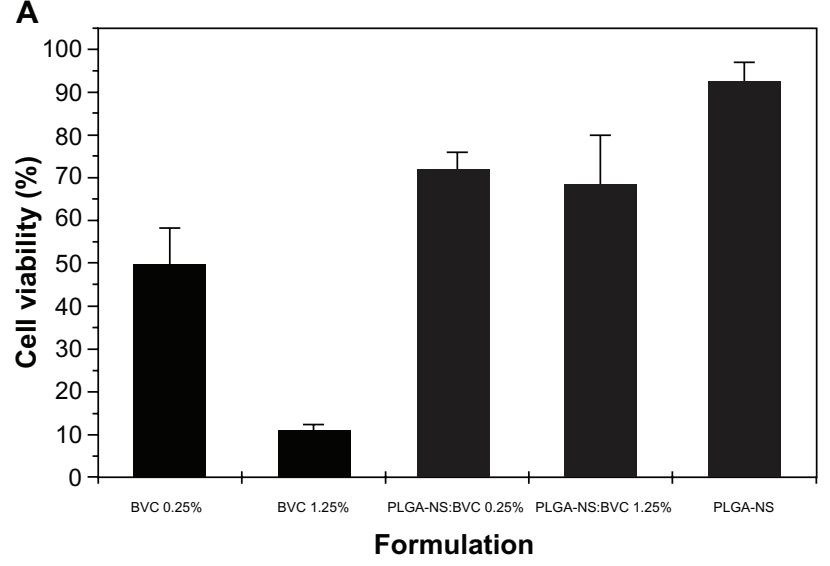

B

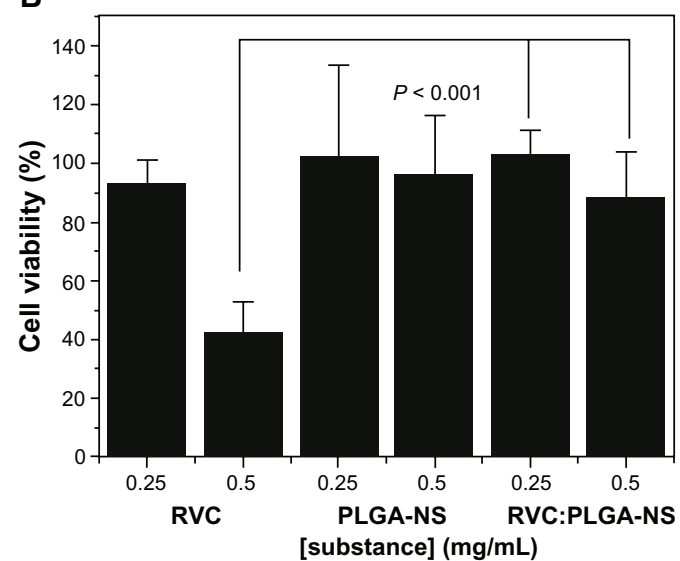

Figure 2 (A) Cytotoxic effects of bupivacaine (BVC), poly(DL-lactide-co-glycolide) nanospheres (PLGA-NSs), and PLGA-NS:bupivacaine at 0.25\% and I.25\% on Balb/c $3 \mathrm{~T} 3$ cells incubated for 24 hours at $37^{\circ} \mathrm{C}$ and $5 \% \mathrm{CO}_{2}$, as evaluated by MTT reduction test ${ }^{17}$ and (B) cytotoxic effects of ropivacaine (RVC), PLGA-NS, and PLGANS:ropivacaine at 0.25 and $1.25 \mathrm{mg} / \mathrm{mL}$ on Balb/c $3 \mathrm{~T} 3$ cells incubated for 24 hours at $37^{\circ} \mathrm{C}$ and $5 \% \mathrm{CO}_{2}$, as evaluated by MTT reduction test. ${ }^{18}$ 

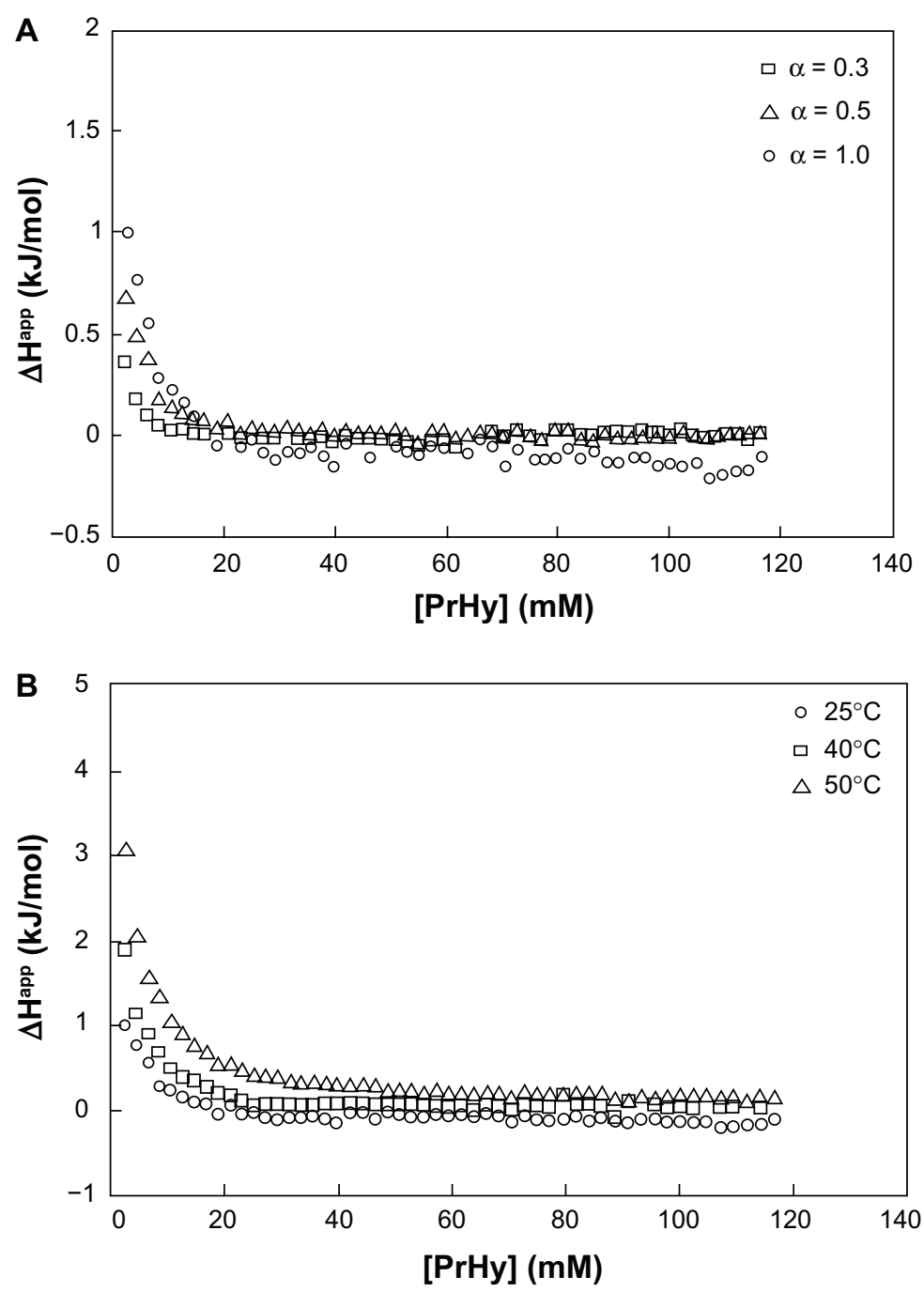

Figure 3 (A) Differential enthalpy curves for titrating $600 \mathrm{mM}$ procaine hydrochloride (PrHy) into 0.1 wt\% HASE 20-80-I at varying degrees of neutralization: $\alpha=0.3$ ( $\square$ ), $\alpha=0.5(\Delta)$, and $\alpha=\mathrm{I}(\diamond)$ and $(\mathbf{B})$ differential enthalpy curves for titrating $600 \mathrm{mM}$ PrHy into fully neutralized $(\alpha=\mathrm{I}) 0 . \mathrm{I}$ wt\% HASE 20-80-I at varying temperatures: $25^{\circ} \mathrm{C}(\diamond), 40^{\circ} \mathrm{C}(\square)$, and $50^{\circ} \mathrm{C}(\Delta) .^{20}$

the release profiles and the effective therapeutic range of drug carriers. Therefore, it is possible to design a drug delivery system with enhanced interaction in order to have a better control of release behavior. ${ }^{20}$

\section{Size effect on the release of drugs}

Histological studies showed that particles with a size of $100 \mathrm{~nm}$ could diffuse through the submucosal layers, whereas larger-sized particles $(500 \mathrm{~nm}-10 \mu \mathrm{m})$ were found to concentrate within the epithelial tissue linings. ${ }^{67}$ Due to the high surface area-to-volume ratio, the release of hydrophilic LA will be rapid, that is indicated by the initial burst release. High initial burst release has been observed for poly(DL-lactide-co-glycolide) nanopaticles, ${ }^{19,68}$ chitosan nanoparticles, ${ }^{69}$ and PNIPAM-co-acrylic acid hydrogels in the submicron range. ${ }^{70} \mathrm{~A}$ simple approach in addressing the problem of initial burst of nanogels was proposed by our group using the layer-by-layer (LBL) technique..$^{71} \mathrm{LBL}$ is a simple technique of coating alternating layers of oppositely charged polyelectrolyte onto a surface. However, the coating must not be too stable, as this will impact the effectiveness of the coated particles as a vehicle for drug delivery. Permeability will be reduced if the coating is too stable, resulting in a continuous slow release of the drugs. ${ }^{71}$ With more polyelectrolyte layers, the more accessible PrHy molecules have to diffuse through the polyethylene (PE) layers, therefore mitigating the burst release phenomenon (Figure 5). ${ }^{71}$

\section{Effect of drug-loading content on drug release}

From the work of Görner et al, ${ }^{6}$ it is shown that size and drug loading do have an effect on the mass of drugs released per milligram 


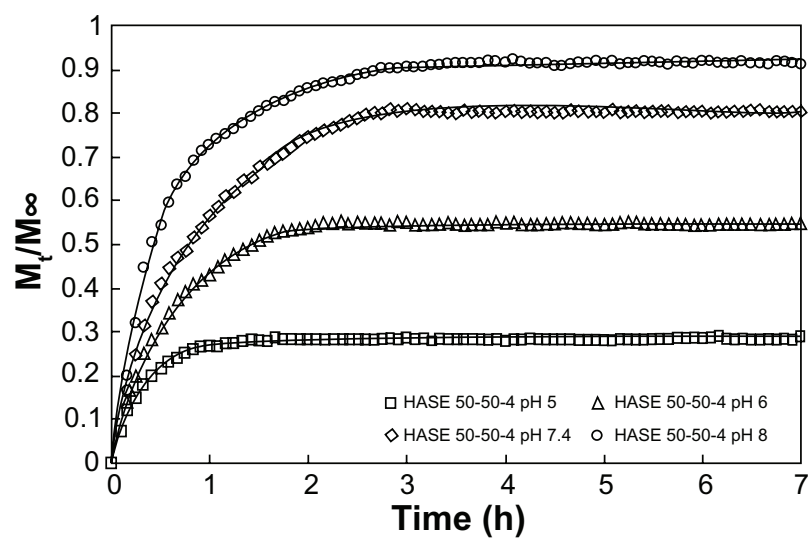

Figure 4 In vitro release profile of procaine hydrochloride from 0.1 wt\% HASE 50-50-4 at varying $\mathrm{pH}: \mathrm{pH} 5(\square), \mathrm{pH} 6(\Delta), \mathrm{pH} 7(\diamond)$, and $\mathrm{pH} 8(\bigcirc) .{ }^{20}$

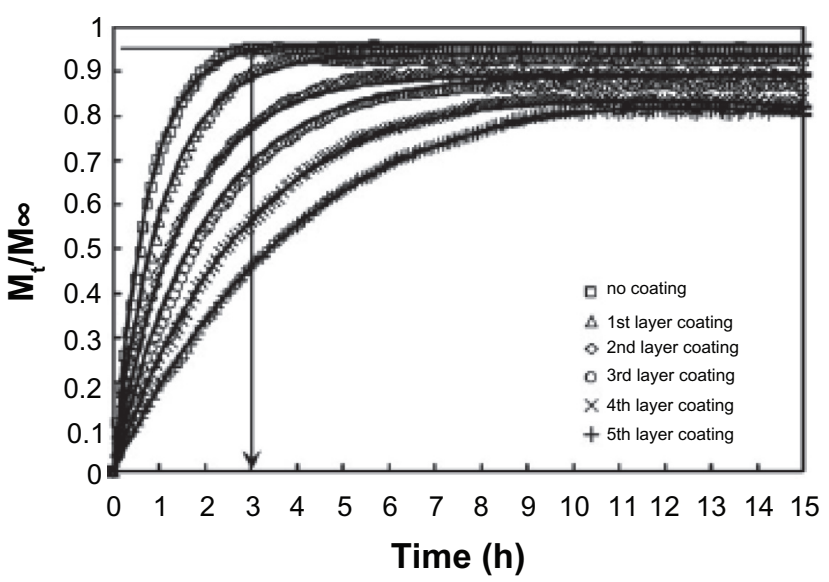

Figure 5 Experimental release profiles for 0.1 wt\% HASE 50-50-4 grafted with PEGMA and polyelectrolyte-coated HASE 50-50-4 grafted with PEGMA nanogels in $100 \mathrm{~mL}$ of $10 \mathrm{mM} \mathrm{NaCl}$ at $\mathrm{pH} 7.4$ (symbols) and theoretical fit of the mathematical model taking into account drug diffusion and chain relaxation (solid lines). ${ }^{71}$

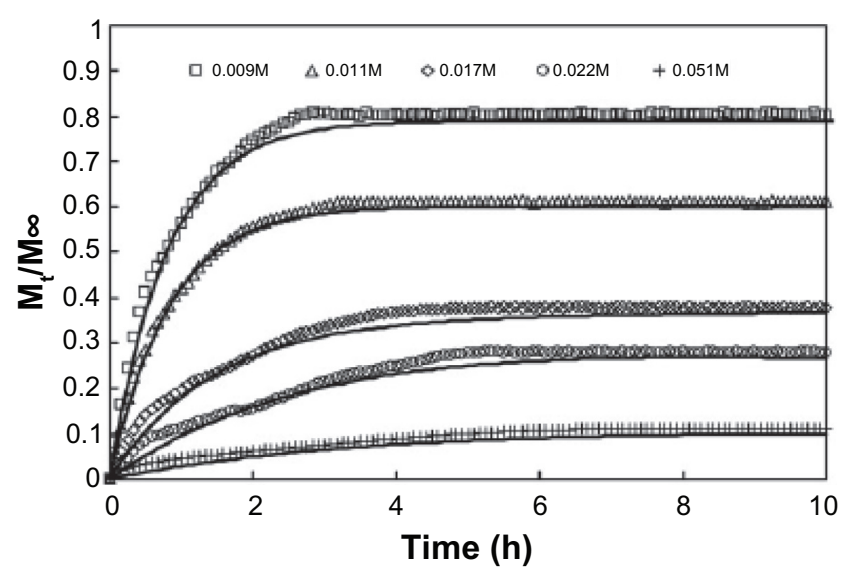

Figure 6 Experimental in vitro release profile of procaine hydrochloride from 0.1 wt\% HASE 50-50-4 at varying initial drug loading: $0.009 M(\square), 0.011 M(\Delta)$, $0.017 \mathrm{M}(\diamond), 0.022 \mathrm{M}(\bigcirc)$, and $0.05 \mathrm{I} M(+)$ and theoretical fit of the mathematical model taking into account drug diffusion and chain relaxation (solid lines) ${ }^{66}$ of nanospheres and the time to attain equilibrium release. Larger particles will result in higher mass of drugs released and longer time to achieve equilibrium. Tan et $\mathrm{al}^{66}$ have also shown that with a higher drug loading content of PrHy, the rate and amount of drugs released will be greatly affected (Figure 6). As a result, we have learned that the drug release rate of the encapsulated drugs is highly dependent on the state (crystalline or dispersed).

\section{Conclusion}

The use of LA could be limited by the relatively short therapeutic action and systemic toxicity related to high drug plasma concentration as a result of fast systemic uptake. Improvement of regional administration of LAs could be achieved by incorporating them into drug delivery systems. Nanogels are probably one of the better candidates due to the lesser pain during injection and longer blood circulation time. However, designing a perfect candidate would require one to have a thorough knowledge of the interaction between the drug and the carrier and the effect of size and drug loading on drug release.

\section{Disclosure}

The authors report no conflict of interest in this work.

\section{References}

1. Bruguerolle B, Labrecque G. Rhythmic pattern in pain and their chronotherapy. Adv Drug Deliv Rev. 2007;59:883-895.

2. Bourne E, Wright C, Royse C. A review of local anesthetic cardiotoxicity and treatment with lipid emulsion. Local Reg Anesth. 2010; 3:11-19.

3. Albright G. Cardiac arrest following regional anesthesia with etidocaine or bupivacaine. Anesthesiology. 1979;51:285-287.

4. Trachez MM, Zapata-Sudo G, Moreira OR, et al. Motor nerve blockade potency and toxicity of non-racemic bupivacaine in rats. Acta Anaesthesiol Scand. 2005;49:66-71.

5. Zapata-Sudo G, Trachez MM, Sudo RT, Nelson TE. Is comparative cardiotoxicity of $\mathrm{S}(-)$ and $\mathrm{R}(+)$ bupivacaine related to enantiomerselective inhibition of L-type $\mathrm{Ca}(2+)$ channels? Anesth Analog. 2001;92: 496-501.

6. Görner T, Gref R, Michenot D, Sommer F, Tran MN, Dellacherie E. Lidocaine-loaded biodegradable nanospheres. I. Optimization of the drug incorporation into the polymer matrix. J Control Release. 1999; $57: 259-268$

7. Soppimath KS, Aminabhavi TM, Kulkarni AR, Dudziski WE. Biodegradable polymeric nanoparticles as drug delivery devices. $J$ Control Release. 2001;70:1-20.

8. Weinberg GL, VadeBoncouer T, Ramaraju GA, Garcia-Amaro MF, Cwik MJ. Pretreatment or resuscitation with a lipid infusion shifts the dose-response to bupivacaine-induced asystole in rats. Anesthesiology. 1998;88:1071-1075.

9. Weinberg G, Ripper R, Feinstein DL, Hoffman W. Lipid emulsion infusion rescues dogs from bupivacaine-induced cardiac toxicity. Reg Anesth Pain Med. 2003;28:198-202.

10. Pinto LMA, Yokaichiya DK, Fraceto LF, de Paula E. Interaction of benzocaine with model membranes. Biophys Chem. 2000;87:213-223.

11. Kohane DS, Lipp M, Kinney RC, Lotan N, Langer R. Sciatic nerve blockade with lipid-protein-sugar particles containing bupivacaine. Pharm Res. 2000;17:1243-1249. 
12. Pinto LMA, Fraceto LF, Santana MHA, Pertinhez TA, Junior SO, de Paula E. Physico-chemical characterization of benzocaine- $\beta$ cyclodextrin inclusion complexes. J Pharm Biomed Anal. 2005;39: 956-963.

13. Blanco MD, Bernardo MV, Sastre RL, Olmo R, Muniz E, Teijón JM. Preparation of bupivacaine-loaded poly( $\varepsilon$-caprolactone) microspheres by spray drying: drug release studies and biocompatibility. Eur J Pharm Biopharm. 2003;55:229-236.

14. Corre PL, Estèbe JP, Clément R, et al. Spray-dryed bupivavaine-loaded microspheres: in vitro eveluation and biopharmaceutics of bupivacaine following brachial plexus adminintration in sheep. Int J Phram. 2002; 238:191-203.

15. Tomoda K, Asahiyama M, Ohtsuki E, et al. Preparation and properties of carrageenan microspheres containing allopurinol and local anesthetic agents for the treatment of oral mucositis. Colloids Surf B Biointerfaces. 2009;71:27-35.

16. Bernardo MV, Blanco MD, Olmo R, Teijón JM. Delivery of bupivacaine included in poly(acrylamide-co-monomethyl itaconate) hydrogels as a function of the $\mathrm{pH}$ swelling medium. J Appl Polym Sci. 2002;86: 327-334.

17. Moraes CM, de Lima R, Rosa AH, de Paula E, Fraceto LF. Encapsulation of local anesthetic bupivacaine in biodegrable poly (DL-lactide-coglycolide) nanospheres: factorial design, characterization and cytotoxicity studies. Macromol Symp. 2009;281:106-112.

18. Moraes CM, de Matos AP, de Lima R, Rosa AH, de Paula E, Fraceto LF. Initial development and characterization of PLGA nanospheres containing ropivacaine. J Biol Phys. 2007;33:455-461.

19. Govender T, Stolnik S, Garnett MC, Illum L, Davis SS. PLGA nanoparticles prepared by nanoprecipitation: drug loading and release studies of a water soluble drug. J Control Release. 1999;57:171-185.

20. Tan JPK, Goh CH, Tam KC. Comparative drug release studies of two cationic drugs from pH-responsive nanogels. Eur J Pharm Sci. 2007; 32:340-348.

21. Kumar MNVR, Kumar N, Domb AJ, Arora M. Pharmaceutical polymeric controlled drug delivery systems. Adv Polym Sci. 2002;160:45-117.

22. Nayak S, Lyon LA. Soft nanotechnology with soft nanoparticles. Angew Chem Int Ed Engl. 2005;44:7686-7708.

23. Donini C, Robinson DN, Colombo P, Giordano F, Peppas NA. Preparation of poly(methacrylic acid-g-poly(ethylene glycol)) nanospheres from methacrylic monomers for pharmaceutical applications. Int $J$ Pharm. 2002;245:83-91.

24. Pelton RH. Temperature-sensitive aqueous microgels. Adv Colloid Interface Sci. 2000;85:1-33.

25. Pelton RH, Chibante P. Preparation of aqueous lattices with N-isopropylacrylamide. Colloids Surf. 1986;20:247-256.

26. Landfester K, Willert M, Antonietti M. Preparation of polymer particles in nonaqueous direct and inverse miniemulsions. Macromolecules. 2000;33:2370-2376

27. Kriwet B, Walter E, Kissel T. Synthesis of bioadhesive poly(acrylic acid) nano- and microparticles using an inverse emulsion polymerization method for the entrapment of hydrophilic drug candidates. J Control Release. 1998;56:149-158.

28. Sun Q, Deng Y. In situ synthesis of temperature-sensitive hollow microspheres via interfacial polymerization. J Am Chem Soc. 2005; $127: 8274-8275$.

29. Neyret S, Vincent B. The properties of polyampholyte microgel particles prepared by microemulsion polymerization. Polymer. 1997;38: 6129-6134.

30. Liu J, Zhang Q, Remsen EE, Wooley KL. Nanostructured materials designed for cell binding and transduction. Biomacromolecules. 2001; 2:362-368

31. Marie E, Rothe R, Antonietti M, Landfester K. Synthesis of polyaniline particles via inverse and direct miniemulsion. Macromolecules. 2003; 36:3967-3973.

32. Murthy N, Xu M, Schuck S, Kunisawa J, Shastri N, Frechet JM. A macromolecular delivery vehicle for protein-based vaccines: acid-degradable proteinloaded microgels. Proc Natl Acad Sci U S A. 2003; 100:4995-5000.
33. Goh SL, Murthy N, Xu M, Frechet JM. Cross-linked microparticles as carriers for the delivery of plasmid DNA for vaccine development. Bioconjug Chem. 2004;15:467-474.

34. Kwon YJ, Standley SM, Goh SL, Frechet JM. Enhanced antigen presentation and immunostimulation of dendritic cells using acid-degradable cationic nanoparticles. J Control Release. 2005;105: 199-212.

35. Oh JK, Tang C, Gao H, Tsarevsky NV, Matyjaszewski K. Inverse miniemulsion ATRP: a new method for synthesis and functionalization of well-defined water-soluble/cross-linked polymeric particles. $J \mathrm{Am}$ Chem Soc. 2006;128:5578-5584.

36. Oh JK, Siegwart DJ, Lee HI, et al. Biodegradable nanogels prepared by atom transfer radical polymerization as potential drug delivery carriers: synthesis, biodegradation, in vitro release, and bioconjugation. $J \mathrm{Am}$ Chem Soc. 2007;129:5939-5945.

37. Booth C, Attwood D. Effects of block architecture and composition on the association properties of poly(oxyalkylene) copolymers in aqueous solution. Macromol Rapid Commun. 2000;21:501-527.

38. Riess G. Micellization of block copolymers. Prog Polym Sci. 2003;28: 1107-1170.

39. Zamurovic M, Christodoulou S, Vazaios A, Iatrou E, Pitsikalis M, Hadjichristidis N. Micellization behavior of complex comblike block copolymer architectures. Macromolecules. 2007;40:5835-5849.

40. Castro E, Barbosa S, Juarez J, Taboada P, Katime IA, Mosquera V. Influence of external factors on the micellization process and aggregate structure of poly(oxy)styrene-poly(oxy)ethylene block copolymers. J Phys Chem B. 2008;112:5296-5304.

41. Denkova AG, Mendes E, Coppens MO. Effects of salts and ethanol on the population and morphology of triblock copolymer micelles in solution. J Phys Chem B. 2008;112:793-801.

42. Bang J, Viswanathan K, Lodge TP, Park MJ, Char K. Temperaturedependent micellar structures in poly(styrene-b-isoprene) diblock copolymer solutions near the critical micelle temperature. JChem Phys. 2004;121:11489-11500.

43. Jansson J, Schillen K, Olofsson G, da Silva RC, Loh W. The interaction between PEO-PPO-PEO triblock copolymers and ionic surfactants in aqueous solution studied using light scattering and calorimetry. $J$ Phys Chem B. 2004;108:82-92.

44. Lin YN, Alexandridis P. Cosolvent effects on the micellization of an amphiphilic siloxane graft copolymer in aqueous solutions. Langmuir. 2002; 18:4220-4231.

45. Hennink WE, van Nostrum CF. Novel crosslinking methods to design hydrogels Adv Drug Deliv Rev. 2002;54:13-36.

46. Vinogradov SV, Batrakova EV, Kabanov AV. Poly(ethylene glycol)-polyethyleneimine nanogel particles: novel drug delivery systems for antisense oligonucleotides. Colloids Surf B. 1999;16:291-304.

47. Sun HW, Yu JH, Gong PJ, et al. Novel core-shell magnetic nanogels synthesized in an emulsion-free aqueous system under UV irradiation for potential targeted radiopharmaceutical applications. Int J Nanosci. 2006;5:253-258.

48. Xu DM, Yao SD, Liu YB, et al. Size-dependent properties of M-PEIs nanogels for gene delivery in cancer cells. Int J Pharm. 2007;338:291-296.

49. Gupta M, Gupta AK. In vitro cytotoxicity studies of hydrogel pullulan nanoparticles prepared by aot/n-hexane micellar system. J Pharm Pharm Sci. 2004;7:38-46.

50. Gupta M, Gupta AK. Hydrogel pullulan nanoparticles encapsulating pBUDLacZ plasmid as an efficient gene delivery carrier. J Control Release. 2004;99:157-166.

51. Kohli E, Han HY, Zeman AD, Vinogradov SV. Formulations of biodegradable nanogel carriers with 5'-triphosphates of nucleoside analogs that display a reduced cytotoxicity and enhanced drug activity. J Control Release. 2007;121:19-27.

52. Lee H, Mok H, Lee S, Oh YK, Park TG. Target-specific intracellular delivery of siRNA using degradable hyaluronic acid nanogels. J Control Release. 2007;119:245-252. 
53. Mok H, Park TG. PEG-assisted DNA solubilization in organic solvents for preparing cytosol specifically degradable PEG/DNA nanogels. Bioconjug Chem. 2006;17:1369-1372.

54. Ma QG, Remsen EE, Kowalewski T, Wooley KL. Two-dimensional, shell-cross-linked nanoparticle arrays. J Am Chem Soc. 2001;123: 4627-4628.

55. Pochan DJ, Chen Z, Cui H, Hales K, Qi K, Wooley KL. Toroidal triblock copolymer assemblies. Science. 2004;306:94-97.

56. Chen Z, Cui H, Hales K, et al. Unique toroidal morphology from composition and sequence control of triblock copolymers. J Am Chem Soc. 2005;127:8592-8593.

57. Harrisson S, Wooley KL. Shell-crosslinked nanostructures from amphiphilic $\mathrm{AB}$ and ABA block copolymers of styrene-alt-(maleic anhydride) and styrene: polymerization, assembly and stabilization in one pot. Chem Commun. 2005;26:3259-3261.

58. Joralemon MJ, O'Reilly RK, Hawker CJ, Wooley KL. Shell clickcrosslinked (SCC) nanoparticles: a new methodology for synthesis and orthogonal functionalization. J Am Chem Soc. 2005;127:16892-16899.

59. Vinogradov SV, Kohli E, Zeman AD. Comparison of nanogel drug carriers and their formulations with nucleoside 5-triphosphates. Pharm Res. 2006;23:920-930.

60. Lee WC, Li YC, Chu IM. Amphiphilic poly(D,L-lactic acid)/ poly(ethylene glycol)/poly(D,L-lactic acid) nanogels for controlled release of hydrophobic drugs. Macromol Biosci. 2006;6:846-854.

61. Rolland JP, Maynor BW, Euliss LE, Exner AE, Denison GM, DeSimone JM. Direct fabrication and harvesting of monodisperse, shapespecific nanobiomaterials. J Am Chem Soc. 2005;127:10096-10100.

62. Gratton SE, Pohlhaus PD, Lee J, Guo J, Cho MJ, DeSimone JM. Nanofabricated particles for engineered drug therapies: a preliminary biodistribution study of PRINT nanoparticles. J Control Release. 2007; 121:10-18.
63. Lee JM, Park KM, Lim SJ, Lee MK, Kim CK. Microemulsion formulation of clonixic acid: solubility enhancement and pain reduction. J Pharm Pharmacol. 2002;54:43-49.

64. Date AA, Nagarsenker MS. Parenteral microemulsions: an overview. Int J Pharm. 2008;355:19-30.

65. Bruce A, Bray D, Lewis J, Raff M, Roberts K, Watson JD. Molecular Biology of the Cell. New York: Garland; 1994.

66. Tan JPK, Zeng AQF, Chang CC, Tam KC. Release kinetics of procaine hydrochloride (PrHy) from $\mathrm{pH}$-responsive nanogels: theory and experiments. Int J Pharm. 2008;357:305-313.

67. Arifin AD, Lee LY, Wang CH. Mathematical modeling and simulation of drug release from microspheres: implications to drug delivery system. Adv Drug Deliv Rev. 2006;58:1274-1325.

68. Lee W, Park J, Yang EH, et al. Investigation of the factors influencing the release rates of cyclosporine A loaded micro- and nanoparticles prepared by high pressure homogenizer. J Control Release. 2002;84:115-123.

69. Borges O, Silva AC, Romeijn SG, et al. Uptake studies rat Payer's patches, cytotoxicity and release studies of alginated coated chitosan nanoparticles for mucosal vaccination. J Control Release. 2006;114: 348-358.

70. Gu J, Xia F, Wu Y, Qu X, Yang Z, Jiang L. Programmable delivery of hydrophilic drug using dually responsive hydrogel cages. J Control Release. 2007;117:396-402.

71. Tan JPK, Wang Q, Tam KC. Control of burst release from nanogels via layer by layer assembly. J Control Release. 2008;128:248-254.
Local and Regional Anesthesia

\section{Publish your work in this journal}

Local and Regional Anesthesia is an international, peer-reviewed, open access journal publishing on the development, pharmacology, delivery and targeting and clinical use of local and regional anesthetics and analgesics. The journal welcomes submitted papers covering original research, basic science, clinical studies, reviews \& evaluations,

\section{Dovepress}

guidelines, expert opinion and commentary, case reports and extended reports. The manuscript management system is completely online and includes a very quick and fair peer-review system, which is all easy to use. Visit http://www.dovepress.com/testimonials.php to read real quotes from published authors. 